\title{
Processing X-ray Fluorescence Tomography Data With XRFtomo
}

Fabricio Marin, Si Chen, Francesco De Carlo, Arthur Glowacki, Olga Antipova and Lu Xi Li

Argonne National Laboratory, Lemont, Illinois, United States

Corresponding author: marinf@anl.gov

X-ray fluorescence imaging coupled with tomography allows scientists to map elemental distributions in three dimensions and recover internal information without physically disturbing a given sample. In addition to an x-ray light source, imaging and positioning instruments for data acquisition, software is needed to perform image registration and reconstruct a 3D image from a set of $2 \mathrm{D}$ projections. To facilitate such 3D data analysis and reconstruction, we have developed a python package named XRFtomo based on an data analysis workflow presented earlier[1]. XRFtomo brings together a variety of image processing tools used for preparing, processing and reconstructing 3D renditions of samples imaged using a synchrotron-based x-ray fluorescence (XRF) microprobe or nanoprobe at the Advanced Photon Source (APS).

The general workflow of XRF tomography involves acquiring a series of 2D projection scans of a sample for a range of sample tilt angles at an XRF beamline. These projections are processed in MAPS[2] or XRF-Maps (a recently developed software for XRF data analysis) for both spectrum fitting and elemental concentration quantification, and exported as hierarchical data format (HDF) files. The tomography dataset is then imported to XRFtomo from which one can select the appropriate data based on fitting or normalization preferences for 3D volume reconstruction. XRFtomo bridges the gap between data acquisition and visualization by providing tools to adjust for misalignment in the imaging process and tools for running reconstructions using TomoPy[3], all with the use of a GUI or command line. The program can output reconstructed cross-sections, projections, sinograms, metadata, and a configuration file with last-used parameters for rapid processing the next time the program is launched. These images serve as inputs for other 3D visualization software for generating volumetric renderings and animations.

We have demonstrated here the tomography reconstruction using XRFtomo on a cluster of human neuroblastoma cells (cell line SK-N-DZ, ATCC CatalogNo.CRL-2149) containing Fe nanoparticles. The dataset was collected at the Bionanoprobe[4] at beamline 9-ID-B of the APS consisting of 48 projections over $141^{\circ}$ angle range. Each projection was $8.2 \mu \mathrm{m} \times 13 \mu \mathrm{m}$ with a $100 \mathrm{~nm}$ pixel size. With XRFtomo, one can easily visualize projections, sinograms (FIG 1, left), and cross-sections (FIG 1, right), and adjust parameters for both alignment and reconstruction for optimization. Volume rendering was performed using Amira, showing the locations of Fe nanoparticles in the cell cluster (FIG 2). The software provides a quick and intuitive way to perform XRF tomography reconstruction and analysis with greater control and flexibility over data processing.

ACKNOWLEDGMENTS: The authors would like to thank Dr. Paunesku from Northwestern University for providing the dataset, and Dr. Ching, Dr. Güursoy, Dr. Vogt, Dr. Jiang, Dr. Lai, and Mr. Roehrig from Argonne National Laboratory for helpful discussions. We thank Mr Vacek from Northwester University for his input on center-finding algorithms. This research used resources of the Advanced Photon Source and the Center for Nanoscale Materials, U.S. Department of Energy (DOE) Office of Science User Facilities operated for the DOE Office of Science by Argonne National Laboratory under Contract No. DE-AC02-06CH11357. 


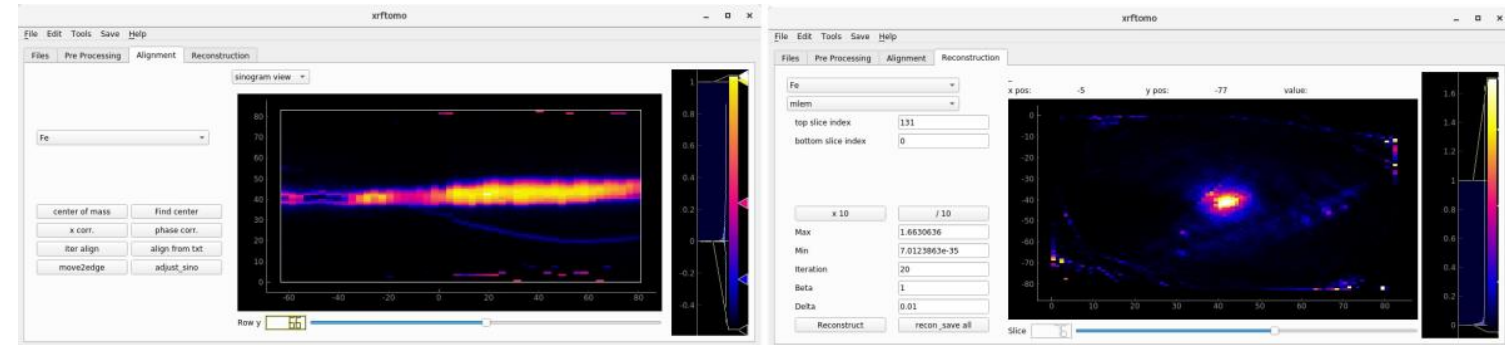

Figure 1. (left) A screenshot of XRFtomo software depicting a sinogram. The sinogram is comprised of one rowof pixels from each projection image oriented vertically for a single element, where the $\mathrm{x}$-axis corresponds to theprojection angle and the y-axis corresponds to pixel location from the projection-view perspective. To the left of theimage are various alignment methods and a dropdown menu to select a different element. (right) A screenshot ofXRFtomo software depicting a reconstructed cross-section of the sample. To the left of the image are reconstructionparameters including the element, method, index range and other method-specific parameters.

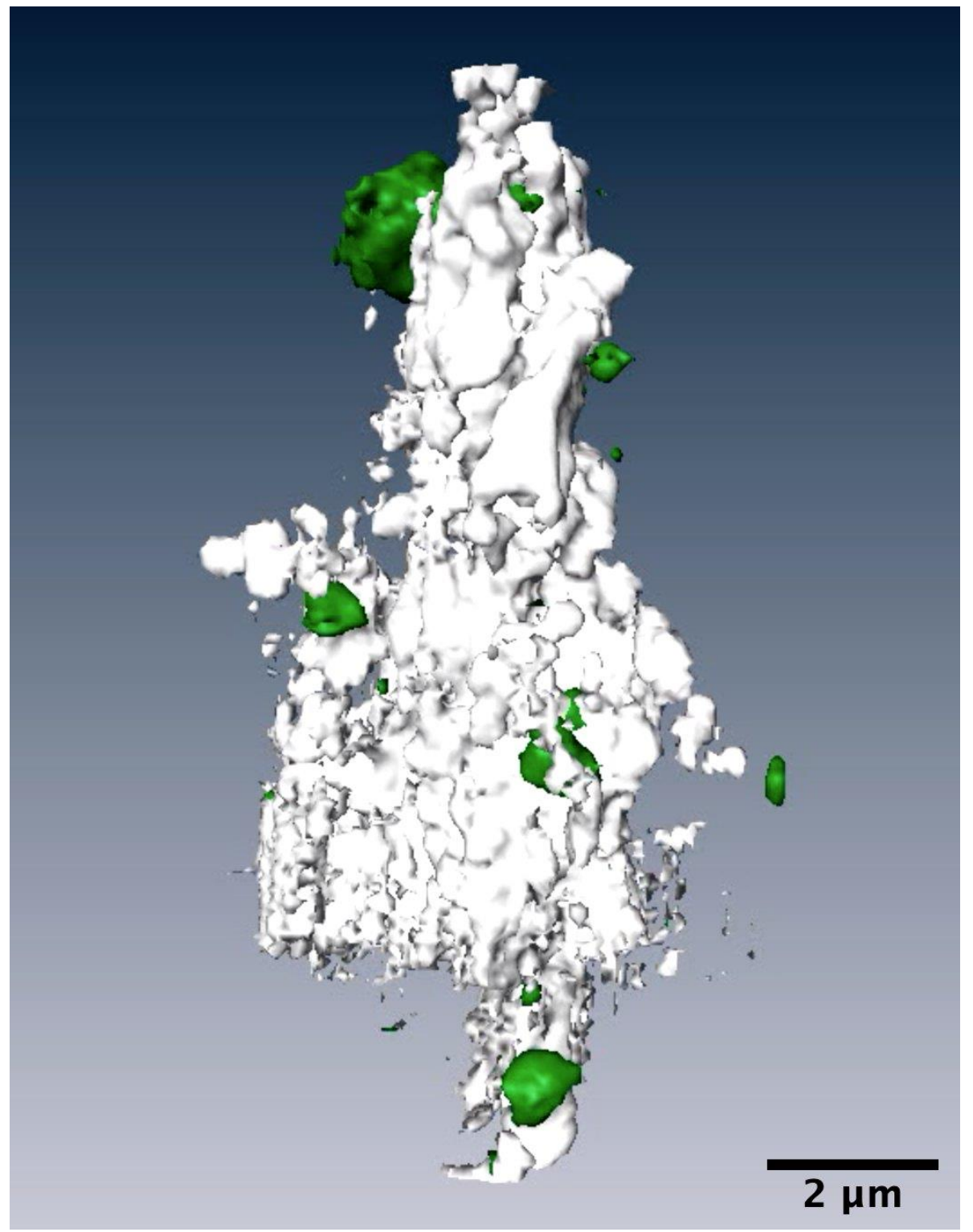


Figure 2. Volume rendering of neuroblastoma cell cluster. $\mathrm{Zn}$ (white) signal is from nuclear materials. Fe (green)signal is from nanoparticles.

\section{References}

[1] Y. P. Hong, S. Chen, and C. Jacobsen, "A new workflow for x-ray fluorescence tomography: MAPStoTomoPy," in X-Ray Nanoimaging: Instruments and Methods II, Vol. 9592, edited by B. Lai, International Society for Optics and Photonics (SPIE, 2015), pp. $120-127$.

[2] S. Vogt, Journal de Physique IV (Proceedings) 104, 635-638March (2003).

[3] D. Gürsoy, F. De Carlo, X. Xiao, and C. Jacobsen, Journal of synchrotron radiation 21, 1188-1193 (2014).

[4] S. Chen, J. Deng, Y. Yuan, C. Flachenecker, R. Mak, B. Hornberger, Q. Jin, D. Shu, B. Lai, J. Maser, C. Roehrig, T. Paunesku, S. C. Gleber, D. J. Vine, L. Finney, J. VonOsinski, M. Bolbat, I. Spink, Z. Chen, J. Steele, D. Trapp, J. Irwin, M. Feser, E. Snyder, K. Brister, C. Jacobsen, G. Woloschak, and S. Vogt, Journal of synchrotron radiation $21,66-75$ (2014). 